\title{
Electronic structure studies of FeSi: A chiral topological system
}

\author{
Susmita Changdar, ${ }^{1}$ S. Aswartham, ${ }^{2}$ Anumita Bose,${ }^{3}$ Y. Kushnirenko, ${ }^{2}$ G. Shipunov, ${ }^{2}$ N. C. Plumb $\odot,{ }^{4}$ M. Shi, ${ }^{4}$ \\ Awadhesh Narayan, ${ }^{3}$ B. Büchner, ${ }^{2}$ and S. Thirupathaiah ${ }^{1,2, *}$ \\ ${ }^{1}$ Condensed Matter Physics and Material Sciences Department, S N Bose National Centre for Basic Sciences, \\ Kolkata, West Bengal 700106, India \\ ${ }^{2}$ Leibniz Institute for Solid State Research, IFW Dresden, D-01171 Dresden, Germany \\ ${ }^{3}$ Solid State and Structural Chemistry Unit, Indian Institute of Science, Bangalore 560012, India \\ ${ }^{4}$ Swiss Light Source, Paul Scherrer Institute, CH-5232 Villigen PSI, Switzerland.
}

(Received 10 December 2019; accepted 13 May 2020; published 1 June 2020)

\begin{abstract}
Most recent observations of topological Fermi arcs on the surface of manyfold degenerate B20 systems, $\mathrm{CoSi}$ and $\mathrm{RhSi}$, have attracted enormous research interests. Although another isostructural system, FeSi, has been predicted to show bulk chiral fermions, it is yet to be clear theoretically and as well experimentally that whether FeSi possesses the topological surface Fermi arcs associated with the exotic chiral fermions in vicinity of the Fermi level. In this contribution, using angle-resolved photoemission spectroscopy (ARPES) and density functional theory (DFT), we present the low-energy electronic structure of FeSi. We further report the surface state calculations to provide insights into the surface band structure of FeSi near the Fermi level. Unlike in CoSi or RhSi, FeSi has no topological Fermi arcs near the Fermi level as confirmed both from ARPES and surface state calculations. Further, the ARPES data show spin-orbit coupling (SOC) band splitting of $40 \mathrm{meV}$, which is in good agreement with bulk band-structure calculations. We noticed an anomalous temperature-dependent resistivity in FeSi which can be understood through the electron-phonon interactions as we find a Debye energy of $80 \mathrm{meV}$ from the ARPES data.
\end{abstract}

DOI: 10.1103/PhysRevB.101.235105

\section{INTRODUCTION}

Since the discovery of linear dispersive Dirac fermions in graphene [1,2], the condensed matter has become fertile ground for the investigation of various exotic topological fermions. Specifically, the experimental realization of threedimensional topological insulators [3] further boosted the field to new heights, from basic sciences [4-27] to more complex technological designs for the futuristic topological quantum computations (TQC) [28-32]. At present, the topological quantum materials are classified by the Weyl fermions [13-25,33], the Dirac fermions [7-12,26], and the Majorana fermions [32,34]. In general, at the band crossing point (BCP), the Weyl fermions have twofold degeneracy and the Dirac fermions have fourfold degeneracy. Recently, new types of quantum materials have emerged with manyfold degenerate fermions at the band crossing point [35-40]. These manyfold degenerate fermions are manifestations of the certain spacegroup symmetries in the presence of the time-reversal invariance [35]. Among them, the chiral systems like the transitionmetal mono-silicides $\mathrm{MSi}(\mathrm{M}=\mathrm{Co}, \mathrm{Mn}, \mathrm{Fe}, \mathrm{Rh})$ have been at the recent intense research focus as under certain conditions, some of these systems (CoSi and $\mathrm{RhSi}$ ) are predicted to show spin-1/2 Weyl fermions with a topological charge of \pm 1 [35-37,39-44], spin-1 excitations with a topological charge of \pm 2 [45], and spin-3/2 Rarita-Schwinger-Weyl (RSW) fermions with topological charges of \pm 4 [46]. Moreover, the

\footnotetext{
*setti@bose.res.in
}

surface Fermi arcs connecting the manyfold degenerate BCPs are much longer in these systems compared to the other known Weyl and Dirac semimetals [19,47-49].

Earlier, the transition metal monosilicides were extensively studied for their low-energy electronic correlations [50-59]. Specifically, FeSi shows peculiar temperature-dependent electronic and magnetic properties [60-65]. Further, FeSi behaves as a semiconductor with an indirect band gap of $50 \mathrm{meV}$ within the temperature range of 100-200 K [57,64], while is a bad metal [66] outside of this temperature range. Different mechanisms were proposed to explain this strange electronic and magnetic behavior, (a) electron-phonon interactions [58,67], (b) spin fluctuations [68,69], and (c) charge excitations [66]. Apart from these interesting physical properties, $\mathrm{FeSi}$ is further predicted to show the above-mentioned manyfold degenerate chiral fermions at the high symmetry points with a nonzero Berry phase [70]. Further, a recent transport study on FeSi shows anomalous temperature-dependent resistivity which they attribute it to the plausible topological surface states [65].

Motivated by the presence of surface Fermi arcs in $\mathrm{RhSi}$ and $\mathrm{CoSi}$, we studied the low-energy electronic structure of isostructural FeSi using angle-resolved photoemission spectroscopy (ARPES) and density functional theory (DFT) to show that despite $\mathrm{FeSi}$ is being chiral topological system, associated surface Fermi arcs connecting the manyfold degenerate bulk BCPs are absent near the Fermi level. These observations are further confirmed by our surface state calculations. The ARPES data clearly show a spin-orbit coupling band splitting of $40 \mathrm{meV}$, consistent with the theoretical 
calculations which predict a spin-orbit coupling (SOC) split of $39.5 \mathrm{meV}$. We further noticed anomalous temperaturedependent resistivity in $\mathrm{FeSi}$, that means, $\mathrm{FeSi}$ is a semiconductor respecting the activation energy formula only within the temperature range of 75-143 K and eventually becoming a bad metal as moving away from this temperature range. The spectral function analysis of the ARPES data suggests an electron-phonon interaction at a Debye energy of $80 \mathrm{meV}$, while the spectral widths near the Fermi level change merely by the thermal excitations within the range of $15-80 \mathrm{~K}$.

\section{EXPERIMENTAL DETAILS}

Single crystals were grown using the floating zone method [71,72]. As grown single crystals were characterized using $\mathrm{x}$-ray diffractometer (XRD) and energy dispersive $\mathrm{x}$-ray analysis (EDAX). These characterization techniques confirm the stoichiometric composition of $\mathrm{FeSi}$ and the space group of $P 2{ }_{1} 3(198)$ [57,73]. More information on XRD and EDAX measurements of FeSi can be found in Ref. [74].

Resistivity measurements were carried out on a closed cycle refrigerator (CCR) based cryostat of CRYOMECH. Four copper $(\mathrm{Cu})$ leads were connected to the sample by vacuum compatible silver epoxy (Epo-Tek H27D) in the Van der Pauw method. The sample temperature was varied between 5 and $310 \mathrm{~K}$ during the measurements.

ARPES measurements were carried out at $1^{3}$-ARPES end station equipped with VG-Scienta R4000 electron analyzer in BESSY II (Helmholtz zentrum Berlin) synchrotron radiation center $[75,76]$. The angular resolution was set at $0.2^{\circ}$ for R4000. Photon energies for the measurements were varied between 30 and $110 \mathrm{eV}$. The energy resolution was set between 10 and $15 \mathrm{meV}$ depending on the excitation energy. Data were recorded at a chamber vacuum of the order of $1 \times$ $10^{-10} \mathrm{mbar}$ and the sample temperature was kept at $1 \mathrm{~K}$ during the measurements. We employed various photon polarizations to extract the electronic structure comprehensively. Another set of ARPES measurements were performed in Swiss Light Source (SLS) at the SIS beamline using a VG-Scienta R4000 electron analyzer. Photon energy was varied between 20 and $120 \mathrm{eV}$. Overall energy resolution was set between 15 and $25 \mathrm{meV}$ depending on the photon energy. Samples were cleaved in situ at a sample temperature of $15 \mathrm{~K}$ and the chamber vacuum was better than $5 \times 10^{-11}$ mbar during the measurements. At SIS beamline, the data were recorded by varying the sample temperature between 15 and $80 \mathrm{~K}$.

\section{BAND-STRUCTURE CALCULATIONS}

Band-structure calculations were performed on the noncentrosymmetric cubic B20 crystal structure of FeSi [77], having the lattice parameters of $a=b=c=4.484 \AA$, using density functional theory (DFT) within the generalized gradient approximation (GGA) of the Perdew, Burke and Ernzerhof (PBE) exchange and correlation potential [78] as implemented in the QUANTUM ESPRESSO simulation package [79]. Ultrasoft nonrelativistic and fully relativistic pseudopotentials were used to perform the calculations without spin-orbit coupling (SOC) and with SOC, respectively. The electronic wave function is expanded using plane waves up to a cutoff energy of

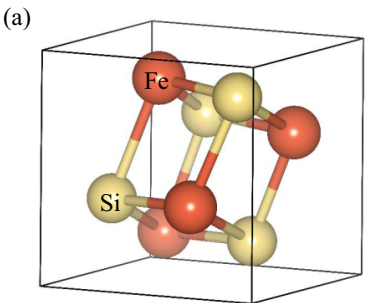

(b)

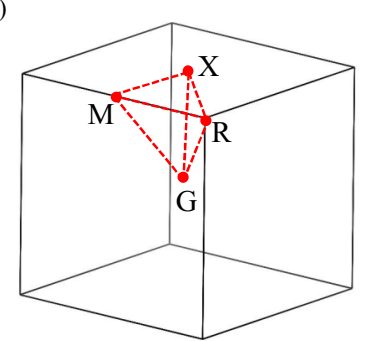

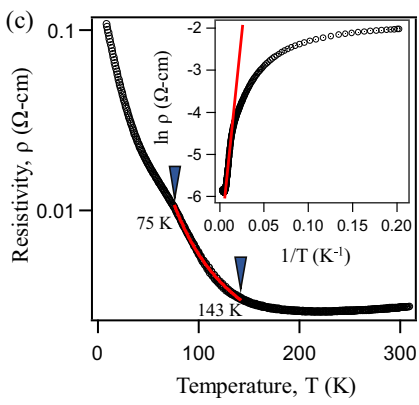

(d)

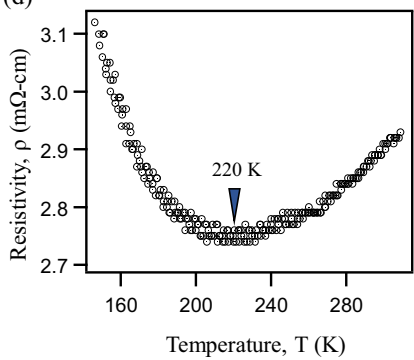

FIG. 1. (a) and (b) are the cubic crystal structure and corresponding Brillouin zone of $\mathrm{FeSi}$, respectively. (c) Temperature-dependent resistivity. Red curve in (c) is the activation formula fitting. Inset in (c) is the $\ln (\rho)$ vs. $1 /$ T. Red line in inset is the linear fitting. (d) Zoomed in resistivity data at high temperature, showing semiconductor to metal cross-over at $T \approx 220 \mathrm{~K}$.

$40 \mathrm{Ry}(545 \mathrm{eV})$. Brillouin zone sampling is done over a $20 \times$ $20 \times 20$ Monkhorst-Pack $k$-grid. The internal coordinates of the system are relaxed before producing the band structure.

For the surface state calculations, the tight-binding model was derived by computing the maximally localized Wannier functions, choosing $\mathrm{Fe} 3 d$ and $\mathrm{Si} 3 p$ orbitals as the basis using the WANNIER90 code [80]. We then employed the WANNIER tools package [81] for analysis of the surface and topological properties. Spin-orbit coupling was included for the surface calculations.

\section{RESULTS AND DISCUSSION}

Resistivity of $\mathrm{FeSi}$ as a function of temperature is shown in Fig. 1. As can be seen from Figs. 1(c) and 1(d), the resistivity of FeSi decreases with increasing temperature up to $220 \mathrm{~K}$, like a semiconductor. However, from a close observation, we realize that $\mathrm{FeSi}$ is a semiconductor only within the temperature range of 75 to $143 \mathrm{~K}$ as it can be properly fitted by the activation formula, $\rho(T)=\rho_{0} e^{\left(\frac{E_{g}}{2 k_{B} T}\right)}$, where $E_{g}$ is the band gap. By fitting the resistivity data, as shown by the red line in the inset of Fig. 1(c), we estimate a semiconducting band gap of $E_{g}=35 \mathrm{meV}$ within this temperature range. The derived gap is in good agreement with the previous report in Ref. [50]. Further, we noticed a kink in the resistivity curve at around $T=75 \mathrm{~K}$, below which $d \rho / d T$ decreases with decreasing temperature when compared to the projected semiconducting nature (see Fig. 5 in Ref. [74]). Similarly, we find that $d \rho / d T$ decreases with increasing temperature between 143 and $220 \mathrm{~K}$. As a result, from our systematic analysis (see Fig. 5 in Ref. [74]), we identified that FeSi is a semiconductor 

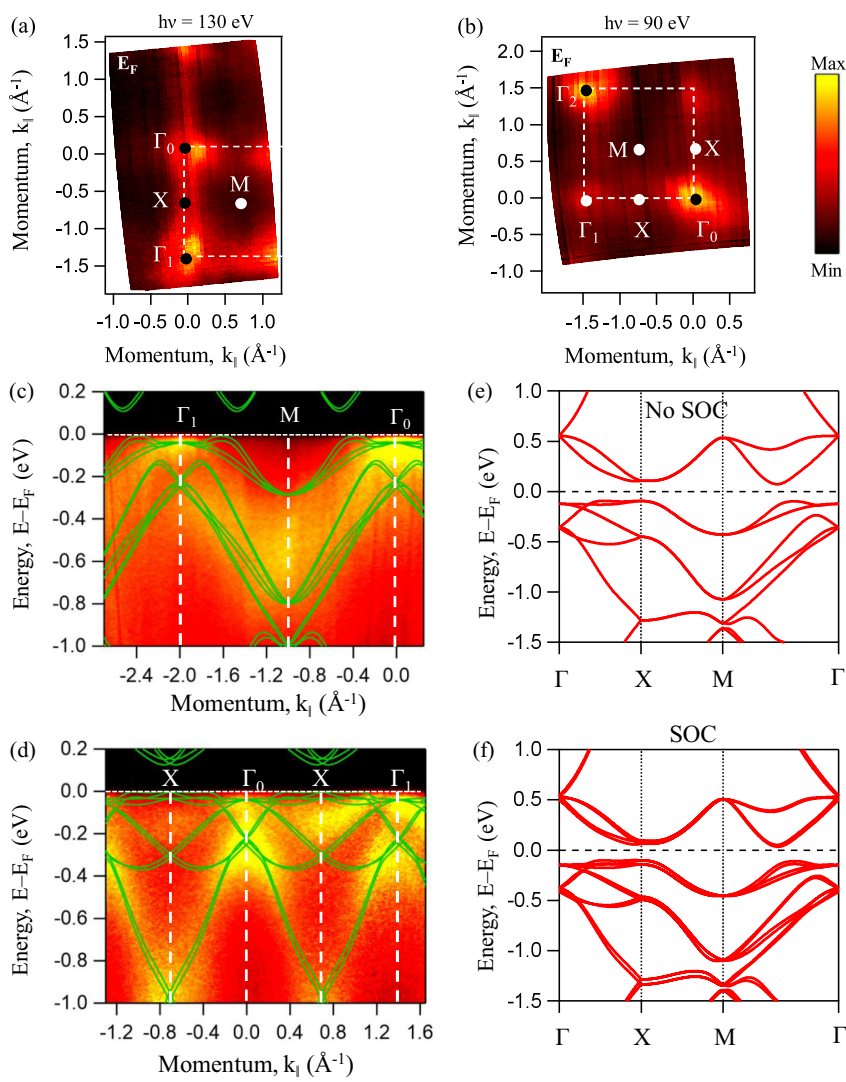

FIG. 2. In-plane electronic band structure of FeSi. (a) and (b) are Fermi surface maps in $k_{x}-k_{y}$ plane measured using photon energy $\mathrm{h} v=130$ and $90 \mathrm{eV}$, respectively. (c) Energy distribution map along the $\Gamma-M$ high symmetry line overlapped with DFT bulk band-structure calculations including SOC. (d) Energy distribution map along the $\Gamma-X$ high symmetry line overlapped with DFT band-structure calculations including SOC. (e) DFT-calculated bandstructure without SOC. (f) DFT-calculated band-structure with SOC.

with a maximum energy gap of $35 \mathrm{meV}$ within the range of 75 to $143 \mathrm{~K}$. Beyond this temperature range, the energy gap of the system rapidly decreases down to a negligible gap of $0.5 \mathrm{meV}$ below $16 \mathrm{~K}$, indicating a bad metal [66]. The same is observed as the system goes above $143 \mathrm{~K}$. And beyond $220 \mathrm{~K}$, the resistivity increases with temperature in a metallic manner. This peculiar resistivity character below $75 \mathrm{~K}$ and above $143 \mathrm{~K}$ can be attributed to bad metallicity of $\mathrm{FeSi}$ $[57,65]$. Thus, our resistivity measurements suggest that $\mathrm{FeSi}$ is a semiconductor following the activation formula within the temperature range of 75 to $143 \mathrm{~K}$ and gradually becomes a bad metal as we go away from this temperature range. These results are qualitatively in agreement with the existing reports, although the semiconducting temperature range is found to be different from different studies [58,65,73,82-85].

Next, ARPES data of FeSi is shown in Fig. 2 recorded at a sample temperature of $1 \mathrm{~K}$. Fermi surface maps in the $k_{x}-k_{y}$ plane are shown in Figs. 2(a) and 2(b) measured using $p$-polarized light with photon energies of 130 and $90 \mathrm{eV}$, respectively. Consistent with the crystal structure, the in-plane Fermi surface (FS) maps show the square symmetry. From the FS maps, we identify a blob-like spectral intensity with fourfold symmetry at the $\Gamma$ point. On the other hand, we did not observe any clear spectral intensity either at the $X$ or $M$ point when measured using $p$-polarized light. To further elucidate the nature of band dispersions, we show energy distribution maps (EDMs) along the high symmetry lines $\Gamma-M$ and $\Gamma-X$ as shown in Figs. 2(c) and 2(d), respectively, measured using $p$-polarized light. DFT bulk band structure including spin-orbit coupling along the respective high symmetry lines is overlapped on to the EDMs. As can be seen from Figs. 2(c) and 2(d), there is a good agreement between the ARPES data and DFT calculations. Note here that the Fermi level of DFT calculations are shifted approximately $100 \mathrm{meV}$ towards the higher binding energy to match with the experimental Fermi level. The band structure from the DFT calculations without SOC and with SOC in the $k$ path $\Gamma X M \Gamma$ are shown in Figs. 2(e) and 2(f), respectively. Further, using $s$-polarized light we could detect flat bands along the $\Gamma-X$ high symmetry line (see Fig. 1 in Ref. [74]), which is in agreement with the DFT calculations. Thus, there is a finite spectral intensity at the $X$ point that is clearly visible with the $s$-polarized light. This suggests that the band structure of $\mathrm{FeSi}$ near the Fermi level is composed of by the multiple orbital characters. More details on the polarization-dependent matrix elements can be found at Ref. [86]. Importantly, in Fig. 2, we did not observe any spectral intensity related to the surface Fermi arcs. Overall, the ARPES data shown in Fig. 2 supports the bad metallic picture of $\mathrm{FeSi}$ at low temperatures as observed from our resistivity measurements. Worth mentioning here that, in Fig. 2, for an easy representation, we did not take into account the $k_{z}$ effects while assigning the high symmetry points on the Fermi surface maps and EDMs.

Photon energy-dependent ARPES data are shown in Fig. 3. Figure 3(a) shows the $k_{y}-k_{z}$ Fermi surface map measured with the photon energies ranging from 75 to $108 \mathrm{eV}$ with a step of $3 \mathrm{eV}$ using $p$-polarized light. The high symmetry points $\Gamma, X$, and $R$ are denoted on the Fermi surface map following the equation $k_{z}=\sqrt{\frac{2 m}{\hbar^{2}}\left(V_{0}+E_{k} \cos ^{2} \theta\right)}$ with an inner potential of $16 \mathrm{eV}$. From the $k_{z}$ Fermi surface map, we realize that the photon energy of $100 \pm 3 \mathrm{eV}$ detects the bands from the $\Gamma$ point and photon energy of $75 \pm 3 \mathrm{eV}$ detects the bands from the $X$ point. Similarly, the high symmetry point $R$ is accessible with a photon energy of $86 \pm 3 \mathrm{eV}$ when the sample surface is normal to the $c$ axis. Energy distribution maps along $\Gamma-X, \Gamma-R$, and $X-R$ are shown in Figs. 3(b), $3(c)$ and 3(d), respectively. The band structure extracted along the in-plane $\Gamma-X([100])$ as shown in Fig. 3(b), is in good agreement with the band structure extracted along the out-ofplane $\Gamma-X$ ([001]) as shown in Fig. 2(d). The band structure derived from DFT calculations with SOC along $\Gamma-R$ and $X-R$ is overlapped on the experimental band structure as shown in Figs. 3(c) and 3(d), and there is a good agreement between DFT calculations and ARPES data. The calculated bulk band structure without SOC and with SOC in the $k$-path $\Gamma R X \Gamma$ are shown in Figs. 3(e) and 3(f), respectively. As predicted from the DFT calculations without SOC, in FeSi the triple-point spin-1 excitations with topological charge of \pm 2 are at the $\Gamma$ point and double Weyl fermions with topological charge of \pm 2 are at the $R$ point. On the other hand, DFT with SOC, the triple-point spin- 1 excitations are predicted at the 

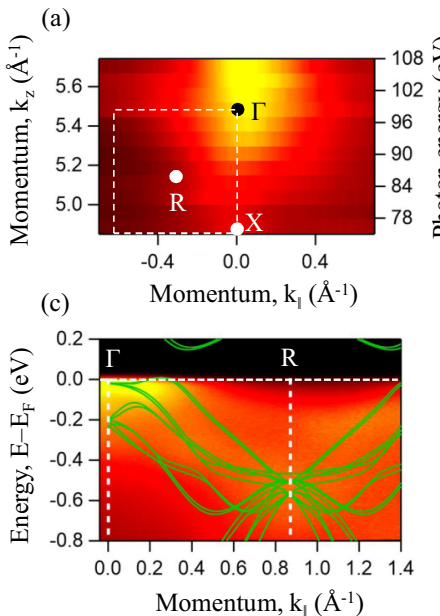

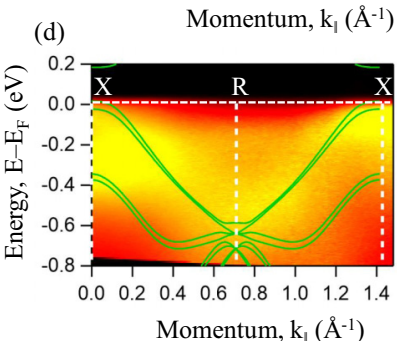

(b)
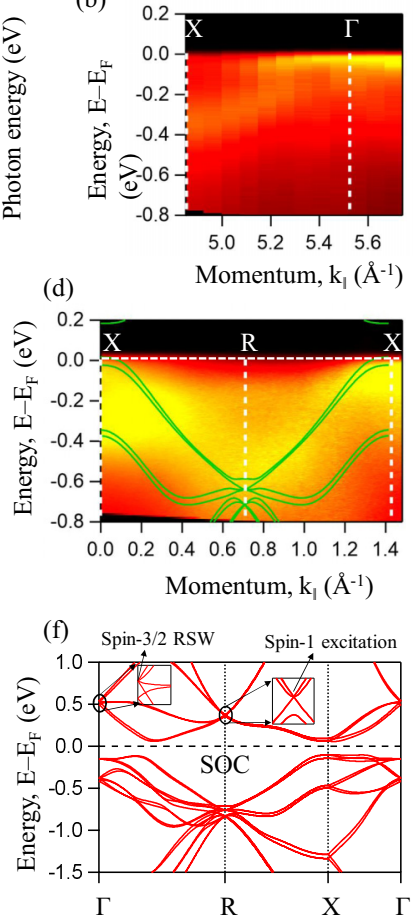

(a)

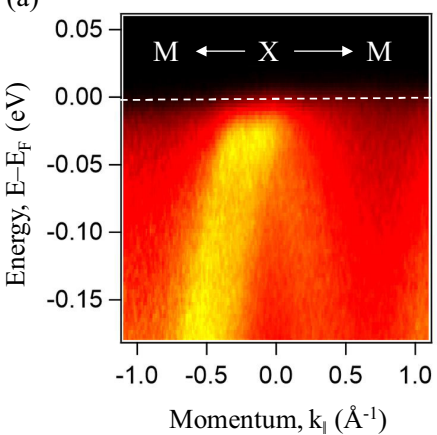

(c)

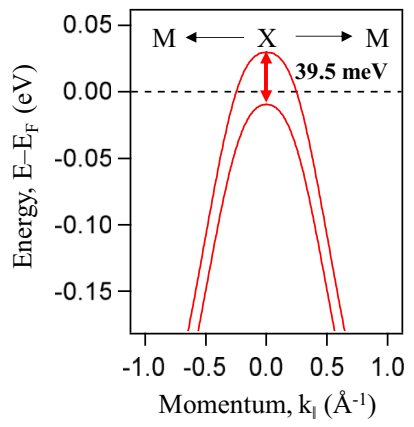

(b)

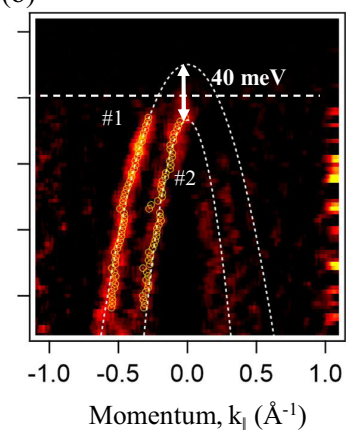

(d)

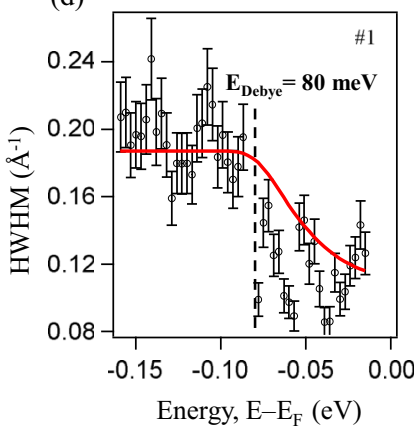

FIG. 3. Out-of-plane electronic band structure of FeSi. (a) Fermi surface map in the $k_{x}-k_{z}$ plane. (b) Energy distribution map along $\Gamma-X$ (001) high symmetry line. (c) Energy distribution map along the $\Gamma-R$ high symmetry line overlapped with DFT band-structure calculated including SOC. (d) Energy distribution map along the $X-R$ high symmetry line overlapped with DFT band-structure calculated including SOC. (e) DFT band structure in the $\Gamma R X \Gamma$ without SOC and (f) with SOC.

$R$ point while the spin-3/2 Rarita-Schwinger-Weyl fermions are predicted at the $\Gamma$ point [36].

Figure 4(a) depicts EDM along the $X-M$ orientation measured at a sample temperature of $15 \mathrm{~K}$. From the second derivative of Fig. 4(a) as shown in Fig. 4(b) we identify two band dispersions, \#1 and \#2. Here, the band \#1 is crossing the Fermi level with a momentum vector of $0.22 \AA^{-1}$ at the $X$ point whereas the band \#2 does not cross the Fermi level. Further, we estimate an energy difference between the top of \#1 and \#2 is about $40 \mathrm{meV}$, which is in good agreement with the DFT calculations with SOC which predicts it to be $39.5 \mathrm{meV}$ as shown in Fig. 4(c). In fact, without SOC there exists only one band dispersion along the $X-M$ orientation at this energy position [see Fig. 2(e)]. Thus, the experimental band structure can be properly reproduced using DFT calculations only with the SOC inclusion. To further elucidate temperature effects on the electronic band structure of FeSi, we measured EDMs along the $X-M$ orientation with temperature ranging between 15 and $80 \mathrm{~K}$ (see Fig. 2 in Ref. [74]). From the temperature-dependent EDMs as shown in Fig. 2 at Ref. [74], it is evident that the band structure near the Fermi level hardly changes with the temperature at least within the range of 15 to $80 \mathrm{~K}$. We further estimated half-width-half-maximum (HWHM) from MDCs, which is directly related to the imaginary part of the self-energy $[\operatorname{Im} \Sigma(E)]$ for the band \#1 as shown in Fig. 4(d). By fitting HWHM using the self-energy
FIG. 4. Temperature-dependent ARPES data. (a) Energy distribution map along the $X-M$ high symmetry line measured using 90-eV photon energies at a sample temperature of $15 \mathrm{~K}$. (b) Second derivative of (a). White dashed curves in (b) are the parabolic fits to the experimental bands. (c) Zoomed in DFT band structure along function of binding energy extracted from the EDM shown in (a) by fitting the momentum dispersive curves (MDCs) with the Lorentzian function. In (d), the red curve is a fit of the self-energy function.

function [87], we find electron-phonon coupling at a Debye energy of $80 \mathrm{meV}$. This estimate of Debye energy is in good agreement with an earlier ARPES report which suggested a Debye energy of $90 \mathrm{meV}$ [54]. Thus, the anomalous resistivity observed in FeSi (see Fig. 1) may not be of the electronic structure origin. But, based on the spectral functional analysis, we suggest that the electron-phonon coupling is playing a crucial role for the observed anomalous resistivity also as suggested by the previous reports [58,67,88,89].

Overall, the experimental band structure is quantitatively in agreement with the DFT calculations. Specifically, the experimental observation of SOC band splitting has been explained very well from DFT calculations with spin-orbit interactions. To date, very few ARPES data with low energy and momentum resolution are available in the literature on $\mathrm{FeSi}$, so it is difficult to compare quantitatively. However, qualitatively, our ARPES data are consistent with some of the earlier ARPES reports [54,55]. Next, coming to the main point of this paper, recent ARPES and theoretical reports on $\mathrm{CoSi}$ and $\mathrm{RhSi}$ showed topological surface Fermi arcs spanned over a large area of two-dimensional (2D) Brillouin zone [36,37,39,40-44,90]. Moreover, they could record manyfold bulk Weyl fermions at $\Gamma$ and $R$ high symmetry points. In contrast, we could not observe any such surface Fermi arcs from our ARPES measurements performed on the $X-M$ orientation. (d) Half width halm maximum (HWHM) as a 

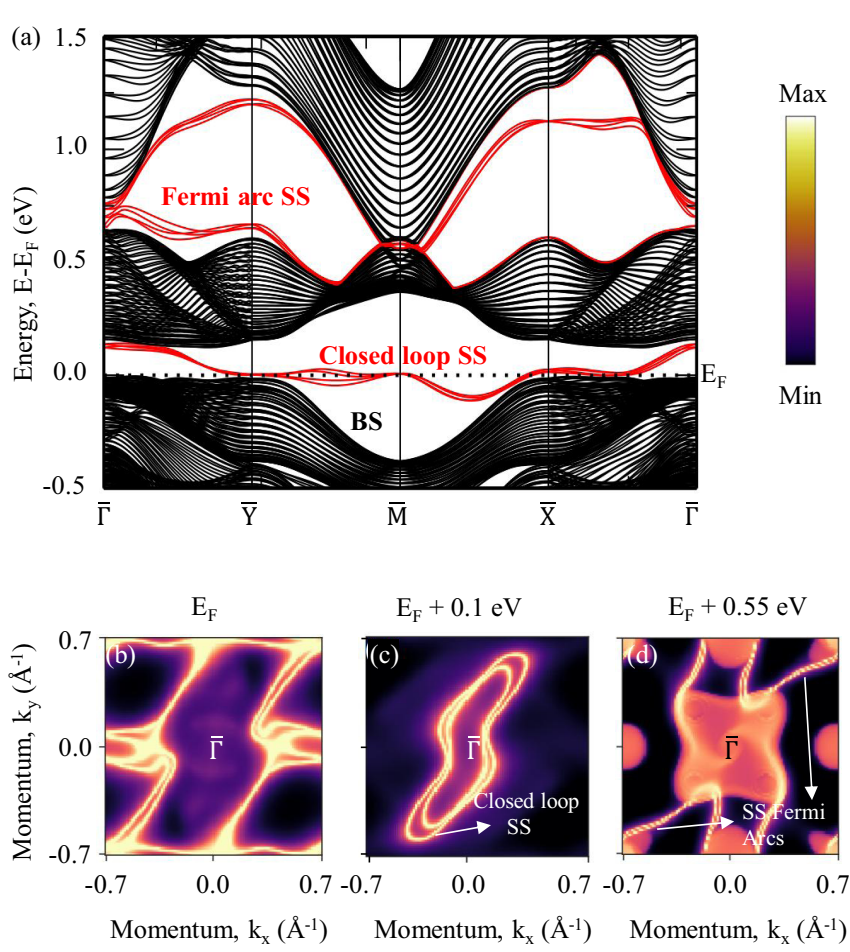

FIG. 5. Band structure calculated for a (001) oriented FeSi slab of thickness $88.96 \AA$. (a) Energy-momentum plot showing surface states (red colored) overlapped with the bulk band structure. Surface state Fermi map taken at (b) $E_{F}$, (c) $E_{F}+0.1 \mathrm{eV}$, and (d) $E_{F}+$ $0.55 \mathrm{eV}$.

isostructural $\mathrm{FeSi}$. As predicted by the DFT calculations [see Fig. 3(f)], in FeSi, the manyfold spin-3/2 RSW fermions are at $0.54 \mathrm{eV}$ and the triple-point spin- 1 excitations are at $0.37 \mathrm{eV}$ above the Fermi level. So it would not be possible to measure them using the ARPES technique. Nevertheless, based on the present understanding, irrespective of the energy position of the manyfold band crossing points (BCPs) one would expect the associated Fermi surface arcs on the surface Brillouin zone $[35,91,92]$.

Therefore, to gain more insight into the surface band structure of $\mathrm{FeSi}$, we carried out density functional theory calculations as shown in Fig. 5. We constructed a WANNIER function based model to compute the band structure of $\mathrm{FeSi}$ slab oriented along the [001] direction. The band structure, for a slab of thickness $88.96 \AA$, along the high symmetry directions is shown in Fig. 5(a). Most noticeably, we find only a set of trivial surface bands within the bulk band gap, and the absence of any topological protected Fermi arcs close to the Fermi level. The topological Fermi arcs, associated with manyfold fermions similar $\mathrm{CoSi}$ and $\mathrm{RhSi}$, occur at substantially higher energies $(0.55 \mathrm{eV}$ above the Fermi level). Furthermore, we also considered the semi-infinite geometry, employing a Green's function method to calculate the surface states, as a function of the in-plane momenta, at different energies. These are presented in Figs. 5(b) to 5(d). In stark contrast to the case of $\mathrm{CoSi}$, we find that these surface states near the Fermi level close-in on themselves as shown in Figs. 5(b) and 5(c), clearly indicating the triviality of these states. The reciprocal space extent of these surface states diminishes as one moves away from the Fermi energy, with the closed loops shrinking in size. However, though the shape of the Fermi arcs is a bit different from $\mathrm{CoSi}$ and $\mathrm{RhSi}$, the nontrivial topological Fermi arcs can be noticed in $\mathrm{FeSi}$ at $0.55 \mathrm{eV}$ above the Fermi level as shown in Fig. 5(d). Thus the surface state calculations indicate that the topological Fermi arcs present in $\mathrm{FeSi}$, however, they are not accessible by conventional ARPES technique. Further, these calculations predicted trivial surface states near the Fermi level which are not well resolved in our ARPES data due to either the surface state spectral intensity is very low compared to the bulk spectral intensity or the sample surface quality is not good enough to detect them.

\section{CONCLUSION}

We systematically studied the low-energy electronic structure of the topological chiral fermionic system, FeSi, using angle-resolved photoemission spectroscopy and density functional theory to derive the following conclusions.

(1) Observation of the Fermi surface from the ARPES measurements suggest that $\mathrm{FeSi}$ is a metal at low temperature, in agreement with our resistivity measurements.

(2) The anomalous temperature-dependent resistivity of FeSi can be explained by the electron-phonon interactions.

(3) ARPES data show a spin-orbit band splitting of $40 \mathrm{meV}$ that is nicely reproduced by the DFT calculations including SOC. Therefore, SOC effects must be considered while discussing the physics of manyfold degenerate fermions in the transition metal monosilicides.

(4) Unlike in the case of $\mathrm{CoSi}$ or $\mathrm{RhSi}, \mathrm{FeSi}$ does not show topological surface Fermi arcs near the Fermi level as the surface state calculations predict them well above the Fermi level. Therefore, we are unable to detect the nontrivial topological surface states in $\mathrm{FeSi}$ experimentally using the conventional ARPES technique.

\section{ACKNOWLEDGMENTS}

S.C. acknowledges University Grants Commission (UGC), India for the PhD fellowship. A.B. thanks the Indian Institute of Science for the PhD fellowship. A.N. acknowledges support from the start up grant (Grant No. SG/MHRD-19-0001) at the Indian Institute of Science. S.A. and B.B. acknowledges financial support by Deutsche Forschungsgemeinschaft (DFG), Germany through the Project No. 419457929. S.A. and B.B. thank DFG through Project No. 405940956. S.T. acknowledges the financial support by DST, India through the INSPIRE-Faculty program (Grant No. IFA14 PH-86). S.T. acknowledges the financial support given by SNBNCBS through the Faculty Seed Grants program. S.T. Acknowledges the travel support given by DST, India (Grant No. SR/NM/Z07/2015) and Jawaharlal Nehru Centre for Advanced Scientific Research (JNCASR) for managing the project. The authors thank Alexander Fedorov and Emile Rienks for their technical support during the experiments performed at BESSYII (HZB). 
[1] K. S. Novoselov, A. K. Geim, S. V. Morozov, D. Jiang, M. I. Katsnelson, I. V. Grigorieva, S. V. Dubonos, and A. A. Firsov, Nature 438, 197 (2005).

[2] A. H. Castro Neto, F. Guinea, N. M. R. Peres, K. S. Novoselov, and A. K. Geim, Rev. Mod. Phys. 81, 109 (2009).

[3] M. Z. Hasan and C. L. Kane, Rev. Mod. Phys. 82, 3045 (2010).

[4] W. Zhang, R. Yu, W. Feng, Y. Yao, H. Weng, X. Dai, and Z. Fang, Phys. Rev. Lett. 106, 156808 (2011).

[5] F. Virot, R. Hayn, M. Richter, and J. van den Brink, Phys. Rev. Lett. 106, 236806 (2011).

[6] B. Yan, L. Müchler, and C. Felser, Phys. Rev. Lett. 109, 116406 (2012).

[7] S. M. Young, S. Zaheer, J. C. Y. Teo, C. L. Kane, E. J. Mele, and A. M. Rappe, Phys. Rev. Lett. 108, 140405 (2012).

[8] Z. Wang, Y. Sun, X.-Q. Chen, C. Franchini, G. Xu, H. Weng, X. Dai, and Z. Fang, Phys. Rev. B 85, 195320 (2012).

[9] Z. Wang, H. Weng, Q. Wu, X. Dai, and Z. Fang, Phys. Rev. B 88, 125427 (2013).

[10] S. Borisenko, Q. Gibson, D. Evtushinsky, V. Zabolotnyy, B. Büchner, and R. J. Cava, Phys. Rev. Lett. 113, 027603 (2014).

[11] M. Neupane, S.-Y. Xu, R. Sankar, N. Alidoust, G. Bian, C. Liu, I. Belopolski, T.-R. Chang, H.-T. Jeng, H. Lin, A. Bansil, F. Chou, and M. Z. Hasan, Nat. Commun. 5, 3786 (2014).

[12] Z. K. Liu, J. Jiang, B. Zhou, Z. J. Wang, Y. Zhang, H. M. Weng, D. Prabhakaran, S.-K. Mo, H. Peng, P. Dudin, T. Kim, M. Hoesch, Z. Fang, X. Dai, Z. X. Shen, D. L. Feng, Z. Hussain, and Y. L. Chen, Nat. Mater. 13, 677 (2014).

[13] X. Huang, L. Zhao, Y. Long, P. Wang, D. Chen, Z. Yang, H. Liang, M. Xue, H. Weng, Z. Fang, X. Dai, and G. Chen, Phys. Rev. X 5, 031023 (2015).

[14] C. Zhang, Z. Yuan, S. Xu, Z. Lin, B. Tong, M. Zahid Hasan, J. Wang, C. Zhang, and S. Jia, Phys. Rev. B 95, 085202 (2017).

[15] N. J. Ghimire, Y. Luo, M. Neupane, D. J. Williams, E. D. Bauer, and F. Ronning, J. Phys.: Condens. Matter 27, 152201 (2015).

[16] C. Shekhar, A. K. Nayak, Y. Sun, M. Schmidt, M. Nicklas, I. Leermakers, U. Zeitler, Y. Skourski, J. Wosnitza, Z. Liu, Y. Chen, W. Schnelle, H. Borrmann, Y. Grin, C. Felser, and B. Yan, Nat. Phys. 11, 645 (2015).

[17] N. Xu, Z. J. Wang, A. P. Weber, A. Magrez, P. Bugnon, H. Berger, C. E. Matt, J. Z. Ma, B. B. Fu, B. Q. Lv, N. C. Plumb, M. Radovic, E. Pomjakushina, K. Conder, T. Qian, J. H. Dil, J. Mesot, H. Ding, and M. Shi, arXiv:1604.02116.

[18] A. Tamai, Q. S. Wu, I. Cucchi, F. Y. Bruno, S. Riccò, T. K. Kim, M. Hoesch, C. Barreteau, E. Giannini, C. Besnard, A. A. Soluyanov, and F. Baumberger, Phys. Rev. X 6, 031021 (2016).

[19] K. Deng, G. Wan, P. Deng, K. Zhang, S. Ding, E. Wang, M. Yan, H. Huang, H. Zhang, Z. Xu, J. Denlinger, A. Fedorov, H. Yang, W. Duan, H. Yao, Y. Wu, S. Fan, H. Zhang, X. Chen, and S. Zhou, Nat. Phys. 12, 1105 (2016).

[20] L. Huang, T. M. McCormick, M. Ochi, Z. Zhao, M.-T. Suzuki, R. Arita, Y. Wu, D. Mou, H. Cao, J. Yan, N. Trivedi, and A. Kaminski, Nat. Mater. 15, 1155 (2016).

[21] A. Liang, J. Huang, S. Nie, Y. Ding, Q. Gao, C. Hu, S. He, Y. Zhang, C. Wang, B. Shen, J. Liu, P. Ai, L. Yu, X. Sun, W. Zhao, S. Lv, D. Liu, C. Li, Y. Zhang, Y. Hu, Y. Xu, L. Zhao, G. Liu, Z. Mao, X. Jia, F. Zhang, S. Zhang, F. Yang, Z. Wang, Q. Peng, H. Weng, X. Dai, Z. Fang, Z. Xu, C. Chen, and X. J. Zhou, arXiv:1604.01706.
[22] Z. Wang, Y. Zheng, Z. Shen, Y. Lu, H. Fang, F. Sheng, Y. Zhou, X. Yang, Y. Li, C. Feng, and Z.-A. Xu, Phys. Rev. B 93, 121112(R) (2016).

[23] J. Jiang, Z. Liu, Y. Sun, H. Yang, C. Rajamathi, Y. Qi, L. Yang, C. Chen, H. Peng, C.-C. Hwang, S. Sun, S.-K. Mo, I. Vobornik, J. Fujii, S. Parkin, C. Felser, B. Yan, and Y. Chen, Nat. Commun. 8, 13973 (2017).

[24] S. Thirupathaiah, R. Jha, B. Pal, J. S. Matias, P. K. Das, P. K. Sivakumar, I. Vobornik, N. C. Plumb, M. Shi, R. A. Ribeiro, and D. D. Sarma, Phys. Rev. B 95, 241105(R) (2017).

[25] S. Thirupathaiah, R. Jha, B. Pal, J. S. Matias, P. K. Das, I. Vobornik, R. A. Ribeiro, and D. D. Sarma, Phys. Rev. B 96, 165149 (2017).

[26] S. Thirupathaiah, I. Morozov, Y. Kushnirenko, A. V. Fedorov, E. Haubold, T. K. Kim, G. Shipunov, A. Maksutova, O. Kataeva, S. Aswartham, B. Büchner, and S. V. Borisenko, Phys. Rev. B 98, 085145 (2018).

[27] G. Chang, B. J. Wieder, F. Schindler, D. S. Sanchez, I. Belopolski, S.-M. Huang, B. Singh, D. Wu, T.-R. Chang, T. Neupert, S.-Y. Xu, H. Lin, and M. Z. Hasan, Nat. Mater. 17, 978 (2018).

[28] C. Nayak, S. H. Simon, A. Stern, M. Freedman, and S. Das Sarma, Rev. Mod. Phys. 80, 1083 (2008).

[29] R. M. Lutchyn, J. D. Sau, and S. Das Sarma, Phys. Rev. Lett. 105, 077001 (2010).

[30] V. Mourik, K. Zuo, S. M. Frolov, S. R. Plissard, E. P. A. M. Bakkers, and L. P. Kouwenhoven, Science 336, 1003 (2012).

[31] A. Stern and N. H. Lindner, Science 339, 1179 (2013).

[32] Introduction to Topological Quantum Matter \& Quantum Computation, edited by T. D. Stanescu, (CRC Press, Boca Raton, FL, 2016), pp. 319-246.

[33] E. Haubold, K. Koepernik, D. Efremov, S. Khim, A. Fedorov, Y. Kushnirenko, J. van den Brink, S. Wurmehl, B. Büchner, T. K. Kim, M. Hoesch, K. Sumida, K. Taguchi, T. Yoshikawa, A. Kimura, T. Okuda, and S. V. Borisenko, Phys. Rev. B 95, 241108(R) (2017).

[34] M. Sato and S. Fujimoto, Phys. Rev. B 79, 094504 (2009).

[35] B. Bradlyn, J. Cano, Z. Wang, M. G. Vergniory, C. Felser, R. J. Cava, and B. A. Bernevig, Science 353, aaf5037 (2016).

[36] P. Tang, Q. Zhou, and S.-C. Zhang, Phys. Rev. Lett. 119, 206402 (2017)

[37] G. Chang, S.-Y. Xu, B. J. Wieder, D. S. Sanchez, S.-M. Huang, I. Belopolski, T.-R. Chang, S. Zhang, A. Bansil, H. Lin, and M. Z. Hasan, Phys. Rev. Lett. 119, 206401 (2017).

[38] D. A. Pshenay-Severin and A. T. Burkov, Materials 12, 2710 (2019).

[39] Z. Rao, H. Li, T. Zhang, S. Tian, C. Li, B. Fu, C. Tang, L. Wang, Z. Li, W. Fan, J. Li, Y. Huang, Z. Liu, Y. Long, C. Fang, H. Weng, Y. Shi, H. Lei, Y. Sun, T. Qian, and H. Ding, Nature 567, 496 (2019).

[40] D. Takane, Z. Wang, S. Souma, K. Nakayama, T. Nakamura, H. Oinuma, Y. Nakata, H. Iwasawa, C. Cacho, T. Kim, K. Horiba, H. Kumigashira, T. Takahashi, Y. Ando, and T. Sato, Phys. Rev. Lett. 122, 076402 (2019).

[41] C. Shekhar, Nat. Mater. 17, 953 (2018).

[42] D. S. Sanchez, I. Belopolski, T. A. Cochran, X. Xu, J.-X. Yin, G. Chang, W. Xie, K. Manna, V. SÃ $1 / 4$ B, C.-Y. Huang, N. Alidoust, D. Multer, S. S. Zhang, N. Shumiya, X. Wang, G.-Q. Wang, T.R. Chang, C. Felser, S.-Y. Xu, S. Jia, H. Lin, and M. Z. Hasan, Nature 567, 500 (2019). 
[43] Y. Yang, H. Sun, J. Xia, H. Xue, Z. Gao, Y. Ge, D. Jia, S. Yuan, Y. Chong, and B. Zhang, Nat. Phys. 15, 645 (2019).

[44] N. B. M. Schröter, D. Pei, M. G. Vergniory, Y. Sun, K. Manna, F. de Juan, J. A. Krieger, V. Süss, M. Schmidt, P. Dudin, B. Bradlyn, T. K. Kim, T. Schmitt, C. Cacho, C. Felser, V. N. Strocov, and Y. Chen, Nat. Phys. 15, 759 (2019).

[45] C. Fang, M. J. Gilbert, X. Dai, and B. A. Bernevig, Phys. Rev. Lett. 108, 266802 (2012).

[46] W. Rarita and J. Schwinger, Phys. Rev. 60, 61 (1941).

[47] B. Q. Lv, H. M. Weng, B. B. Fu, X. P. Wang, H. Miao, J. Ma, P. Richard, X. C. Huang, L. X. Zhao, G. F. Chen, Z. Fang, X. Dai, T. Qian, and H. Ding, Phys. Rev. X 5, 031013 (2015).

[48] Y. Sun, S.-C. Wu, and B. Yan, Phys. Rev. B 92, 115428 (2015).

[49] S.-Y. Xu, I. Belopolski, N. Alidoust, M. Neupane, G. Bian, C. Zhang, R. Sankar, G. Chang, Z. Yuan, C.-C. Lee, S.-M. Huang, H. Zheng, J. Ma, D. S. Sanchez, B. Wang, A. Bansil, F. Chou, P. P. Shibayev, H. Lin, S. Jia, and M. Z. Hasan, Science 349, 613 (2015).

[50] C. Fu, M. P. C. M. Krijn, and S. Doniach, Phys. Rev. B 49, 2219 (1994).

[51] C.-H. Park, Z.-X. Shen, A. G. Loeser, D. S. Dessau, D. G. Mandrus, A. Migliori, J. Sarrao, and Z. Fisk, Phys. Rev. B 52, R16981 (1995).

[52] M. A. Chernikov, L. Degiorgi, E. Felder, S. Paschen, A. D. Bianchi, H. R. Ott, J. L. Sarrao, Z. Fisk, and D. Mandrus, Phys. Rev. B 56, 1366 (1997).

[53] K. Ishizaka, T. Kiss, T. Shimojima, T. Yokoya, T. Togashi, S. Watanabe, C. Q. Zhang, C. T. Chen, Y. Onose, Y. Tokura, and S. Shin, Phys. Rev. B 72, 233202 (2005).

[54] M. Klein, D. Zur, D. Menzel, J. Schoenes, K. Doll, J. Röder, and F. Reinert, Phys. Rev. Lett. 101, 046406 (2008).

[55] M. Arita, K. Shimada, Y. Takeda, M. Nakatake, H. Namatame, M. Taniguchi, H. Negishi, T. Oguchi, T. Saitoh, A. Fujimori, and T. Kanomata, Phys. Rev. B 77, 205117 (2008).

[56] Y. N. Zhao, H. L. Han, Y. Yu, W. H. Xue, and T. Gao, Europhys. Lett. 85, 47005 (2009).

[57] A. E. Petrova, V. N. Krasnorussky, A. A. Shikov, W. M. Yuhasz, T. A. Lograsso, J. C. Lashley, and S. M. Stishov, Phys. Rev. B 82, 155124 (2010).

[58] B. C. Sales, O. Delaire, M. A. McGuire, and A. F. May, Phys. Rev. B 83, 125209 (2011).

[59] P. Dutta and S. K. Pandey, Comput. Condens. Matter 16, e00325 (2018).

[60] V. Jaccarino, G. K. Wertheim, J. H. Wernick, L. R. Walker, and S. Arajs, Phys. Rev. 160, 476 (1967).

[61] Y. Takahashi and T. Moriya, J. Phys. Soc. Jpn. 46, 1451 (1979).

[62] Y. Takahashi, J Phys: Condens Matter 10, L671 (1998).

[63] N. E. Sluchanko, V. V. Glushkov, S. V. Demishev, A. A. Menovsky, L. Weckhuysen, and V. V. Moshchalkov, Phys. Rev. B 65, 064404 (2002).

[64] Z. Schlesinger, Z. Fisk, H.-T. Zhang, M. B. Maple, J. F. DiTusa, and G. Aeppli, Phys. Rev. Lett. 71, 1748 (1993).

[65] Y. Fang, S. Ran, W. Xie, S. Wang, Y. S. Meng, and M. B. Maple, Proc. Natl. Acad. Sci. USA 115, 8558 (2018).

[66] J. M. Tomczak, K. Haule, and G. Kotliar, Proc. Natl. Acad. Sci. 109, 3243 (2012).

[67] O. Delaire, K. Marty, M. B. Stone, P. R. C. Kent, M. S. Lucas, D. L. Abernathy, D. Mandrus, and B. C. Sales, Proc. Natl. Acad. Sci. 108, 4725 (2011).
[68] S. N. Evangelou and D. M. Edwards, J. Phys. C: Solid State Phys. 16, 2121 (1983).

[69] Y. Takahashi, J. Phys.: Condens. Matter 9, 2593 (1997).

[70] J. KÃ¹/4bler, B. Yan, and C. Felser, Europhys. Lett. 104, 30001 (2013).

[71] K. Mason, Prog. Cryst. Growth Charact. 2, 269 (1979).

[72] A. Damascelli, K. Schulte, D. van der Marel, and A. A. Menovsky, Phys. Rev. B 55, R4863 (1997).

[73] B. C. Sales, E. C. Jones, B. C. Chakoumakos, J. A. FernandezBaca, H. E. Harmon, J. W. Sharp, and E. H. Volckmann, Phys. Rev. B 50, 8207 (1994).

[74] See Supplemental Material at http://link.aps.org/supplemental/ 10.1103/PhysRevB.101.235105 for additional ARPES, Resistivty, XRD, and EDAX analysis of FeSi.

[75] S. V. Borisenko, Synchrotron Radiation News 25, 6 (2012).

[76] S. V. Borisenko, V. B. Zabolotnyy, D. V. Evtushinsky, T. K. Kim, I. V. Morozov, A. N. Yaresko, A. A. Kordyuk, G. Behr, A. Vasiliev, R. Follath, and B. Büchner, J. Vis. Exp. 68, e50129 (2012).

[77] B. E. Brown, Acta Crystallogr. 20, 268 (1966).

[78] J. P. Perdew, K. Burke, and M. Ernzerhof, Phys. Rev. Lett. 77, 3865 (1996).

[79] P. Giannozzi, S. Baroni, N. Bonini, M. Calandra, R. Car, C. Cavazzoni, D. Ceresoli, G. L. Chiarotti, M. Cococcioni, I. Dabo, A. Dal Corso, S. de Gironcoli, S. Fabris, G. Fratesi, R. Gebauer, U. Gerstmann, C. Gougoussis, A. Kokalj, M. Lazzeri, L. Martin-Samos, N. Marzari, F. Mauri, R. Mazzarello, S. Paolini, A. Pasquarello, L. Paulatto, C. Sbraccia, S. Scandolo, G. Sclauzero, A. P. Seitsonen, A. Smogunov, P. Umari, and R. M. Wentzcovitch, J. Phys.: Condens. Matter 21, 395502 (2009).

[80] A. A. Mostofi, J. R. Yates, Y.-S. Lee, I. Souza, D. Vanderbilt, and N. Marzari, Comput. Phys. Commun. 178, 685 (2008).

[81] Q. Wu, S. Zhang, H.-F. Song, M. Troyer, and A. A. Soluyanov, Comput. Phys. Commun. 224, 405 (2018).

[82] P. Samuely, P. Szabó, M. Mihalik, N. Hudáková, and A. Menovsky, Physica B 218, 185 (1996).

[83] B. Buschinger, C. Geibel, F. Steglich, D. Mandrus, D. Young, J. Sarrao, and Z. Fisk, Physica B: Condensed Matter 230-232, 784 (1997).

[84] S. Paschen, E. Felder, M. A. Chernikov, L. Degiorgi, H. Schwer, H. R. Ott, D. P. Young, J. L. Sarrao, and Z. Fisk, Phys. Rev. B 56, 12916 (1997).

[85] M. Fäth, J. Aarts, A. A. Menovsky, G. J. Nieuwenhuys, and J. A. Mydosh, Phys. Rev. B 58, 15483 (1998).

[86] S. Thirupathaiah, S. de Jong, R. Ovsyannikov, H. A. Dürr, A. Varykhalov, R. Follath, Y. Huang, R. Huisman, M. S. Golden, Y.-Z. Zhang, H. O. Jeschke, R. Valentí, A. Erb, A. Gloskovskii, and J. Fink, Phys. Rev. B 81, 104512 (2010).

[87] T. Valla, A. V. Fedorov, P. D. Johnson, and S. L. Hulbert, Phys. Rev. Lett. 83, 2085 (1999).

[88] A.-M. Racu, D. Menzel, J. Schoenes, and K. Doll, Phys. Rev. B 76, 115103 (2007).

[89] D. Menzel, P. Popovich, N. N. Kovaleva, J. Schoenes, K. Doll, and A. V. Boris, Phys. Rev. B 79, 165111 (2009).

[90] N. B. M. Schröter, S. Stolz, K. Manna, F. de Juan, M. G. Vergniory, J. A. Krieger1, D. Pei, P. Dudin, T. K. Kim, C. Cacho, B. Bradlyn, H. Borrmann, M. Schmidt, R. Widmer, V. Strokov, and C. Felser, arXiv:1907.08723. 
[91] Y. Sun, S.-C. Wu, M. N. Ali, C. Felser, and B. Yan, Phys. Rev. B 92, 161107(R) (2015).

[92] C. Wang, Y. Zhang, J. Huang, S. Nie, G. Liu, A. Liang, Y. Zhang, B. Shen, J. Liu, C. Hu, Y. Ding, D. Liu, Y. Hu, S. He,
L. Zhao, L. Yu, J. Hu, J. Wei, Z. Mao, Y. Shi, X. Jia, F. Zhang, S. Zhang, F. Yang, Z. Wang, Q. Peng, H. Weng, X. Dai, Z. Fang, Z. Xu, C. Chen, and X. J. Zhou, Phys. Rev. B 94, 241119(R) (2016). 